\title{
Population genomics and climate adaptation of a C4 perennial grass, Panicum hallii (Poaceae)
}

\author{
Billie A. Gould 1,2,3 (D), Juan Diego Palacio-Mejia ${ }^{4}$, Jerry Jenkins ${ }^{5}$, Sujan Mamidi ${ }^{5}$, Kerrie Barry ${ }^{6}$, Jeremy Schmutz ${ }^{5,6}$, \\ Thomas E. Juenger ${ }^{4}$ and David B. Lowry ${ }^{2,3,7^{*}}$
}

\begin{abstract}
Background: Understanding how and why genetic variation is partitioned across geographic space is of fundamental importance to understanding the nature of biological species. How geographical isolation and local adaptation contribute to the formation of ecotypically differentiated groups of plants is just beginning to be understood through population genomic studies. We used whole genome sequencing combined with association study of climate to discover the drivers of differentiation in the perennial C4 grass Panicum hallii.

Results: Sequencing of 89 natural accessions of P.hallii revealed complex population structure across the species range. Major population genomic separation was found between subspecies P.hallii var. hallii and var. filipes as well as between at least four major unrecognized subgroups within var. hallii. At least 139 genomic SNPs were significantly associated with temperature or precipitation across the range and these SNPs were enriched for nonsynonymous substitutions. SNPs associated with temperature and aridity were more often found in or near genes than expected by chance and enriched for putative involvement in dormancy processes, seed maturation, response to hyperosmosis and salinity, abscisic acid metabolism, hormone metabolism, and drought recovery.

Conclusions: Both geography and climate adaptation contribute significantly to patterns of genome-wide variation in P.hallii. Population subgroups within P.hallii may represent early stages in the formation of ecotypes. Climate associated loci identified here represent promising targets for future research in this and other perennial grasses.
\end{abstract}

Keywords: Population genomics, Climate GWAS, Ecotypes, Local adaptation, Grasses

\section{Background}

Understanding how and why genetic variation is partitioned across geographic space is of fundamental importance to understanding the nature of biological species [1-3]. For widespread species, the combination of geography and local adaptation is well known to contribute to differentiation of populations and species across space $[4,5]$. In plants, the evolution of locally adapted ecotypes in distinct geographic regions is common $[2,5,6]$. Those ecogeographically partitioned ecotypes typically constitute genetically structured groups

\footnotetext{
* Correspondence: dlowry@msu.edu

${ }^{2}$ Department of Plant Biology, Michigan State University, East Lansing, Ml 48824, USA

${ }^{3}$ Great Lakes Bioenergy Research Center, Michigan State University, East Lansing, Ml 48824, USA

Full list of author information is available at the end of the article
}

[7], which are reproductively isolated from each other to varying degrees (but see [8]). While general patterns in the partitioning of plant species across space is well established for many species, an understanding of the extent to which different ecotypes overlap in their ranges and the distribution of locally adapted alleles across those groups is just beginning to emerge [7]. Overall, studies of population genomic variation in individual plant species can provide insights into the patterns and drivers of genetic divergence across particular geographic regions that vary in climate.

Hall's panicgrass, Panicum hallii Vasey, is a perennial C4 grass and a member of the Panicoid grasses, which includes sorghum, maize, and sugarcane [9]. P. hallii is a diploid model system for the closely related tetraploid bioenergy crop switchgrass (Panicum virgatum). Beyond 
having a favorable ploidy for genetics, $P$. hallii has a short generation time, a small stature, a moderate sized genome $(\sim 550 \mathrm{Mbp})$, and a primarily self-fertilizing mating system [10, 11]. Just like switchgrass, which is composed of two major ecotypes (upland and lowland [12]), P. hallii has two major ecotypic subspecies (varieties), P. hallii var. hallii (hereafter var. hallii) and P.hallii var. filipes (hereafter var. filipes) [13, 14]. Similar to upland switchgrass, var. hallii is typically found in drier habitats across the range of the species, which stretches from Arizona to central Texas and south into northern Mexico. P. hallii var. filipes, like lowland switchgrass, is typically found in more mesic habitats and is restricted to the southern Rio Grande Valley and the Gulf-Coast of South Texas and Northern Mexico. In general, var. hallii is smaller in stature, has fewer tillers, has larger seeds, and flowers earlier than var. filipes $[12,15]$. The two varieties are cross compatible and quantitative trait locus (QTL) analyses have revealed that large-effect, potentially pleiotropic, QTLs are responsible for morphological divergence between them [15]. Further, the two varieties have distinct patterns of physiological and gene expression responses to soil water availability under field conditions [16].

A recent microsatellite-based population structure analysis by Lowry et al. [11] suggested that var. hallii is further subdivided into two subgroups, one occurring primarily in low elevation regions of Texas, with the other occupying higher elevation sky islands of Chihuahuan and Sonoran deserts. Thus, the population structure of $P$. hallii is likely more complex than the major division between the two recognized varieties. Despite this new insight, the previous study by Lowry et al. [11] utilized only 15 genetic markers and therefore could not distinguish fine-scale structure or identify loci that might have experienced natural selection across the range of $P$. hallii. Further, the previous study only had one accession from northern Mexico, which represents a large portion of $P$. hallii's range, and only three accessions of var. filipes.

In this study, we conducted whole genome resequencing of accessions of $P$. hallii from 89 locations to better understand genomic variation and patterns of population structure and across its range. With whole genome resequencing, we were afforded the opportunity to identify potential candidate genes and genomic regions that could be involved in local adaptation to different ecoregions. Overall, our goals were: 1) To characterize finescale population structure and hybridization between and within P.hallii subgroups; 2) To identify regions of the genome associated with local adaptation to major climate variation in temperature and precipitation; and 3) To provide a genomic resource for future studies into the population genomics of perennial grasses.

\section{Results}

Population divergence and structure

Genome sequencing of $P$. hallii has revealed that much of the large pericentromeric region of the genome consists of a low-recombination region with a high level of repetitive DNA. Such large pericentromeric regions are common across plant species $[17,18]$. To reduce false SNP calls in these regions, we eliminated areas of low sequencing/mapping depth ( $<8$ reads) and unusually high heterozygosity (see Methods). After filtering, we identified 9.2 million probable SNPs across the 89 diverse $P$. hallii accessions. Given that an appreciable rate of erroneous variant calls likely still occurs in the data, we estimated that the upper bound for pairwise diversity $(\pi)$, based on callable sites ( $46 \%$ of the genome), as 0.008 within var. filipes and 0.007 within var. hallii. Further, pairwise divergence $\left(D_{x y}\right)$ was greater for comparisons between var. filipes and any var. hallii groups than between any of the var. hallii groups (Figs. 1,2).

The distribution of SNP variation confirmed a strong population split between var. hallii $(N=78)$ and var. filipes $(N=11)$. Within var. hallii, linkage disequilibrium decayed $\left(r^{2}<=0.2\right)$ at around $6 \mathrm{~kb}$ while within var. filipes, linkage remained high $\left(r^{2}>0.3\right)$ at distances greater than $25 \mathrm{~kb}$ (Additional file 1: Figure S1). We hypothesize that greater linkage disequilibrium in var. filipes may result from a small effective population size and possible historical population bottlenecks. In addition to the predicted divergence between subspecies, analysis with both STRUCTURE and RAxML revealed at least four major groups within var. hallii (Fig. 1, Additional file 1: Figure S2). The four major groups within var. hallii included a widespread group that occupies northwestern Texas westward into New Mexico and Arizona (red), and three more geographically overlapping groups. A small group is restricted to central Texas (blue) that overlaps with a second group that ranges from central Texas south into northern Mexico (yellow). A third var. hallii subgroup occurs in south Texas (purple) and overlaps to a great extent with the range of var. filipes. Interestingly, the degree of divergence between P.hallii var. hallii subgroups (max $\left.D_{x y}=0.008\right)$, which are not formally recognized as subspecies, approached the level of divergence found between recognized subspecies var. filipes and var. hallii $\left(D_{x y}=0.012\right.$, Fig. 2$)$.

We found evidence that hybridization occurs between var. filipes and var. hallii albeit at a relatively low rate. If hybridization between subspecies were common throughout history we would expect many individuals to show evidence of mixed ancestry however only a single individual located in the southern part of the var. filipes range showed evidence of possible admixture between var. filipes and the Mexican var. hallii group (Fig. 1). A sympatric population between var. filipes and var. hallii has 

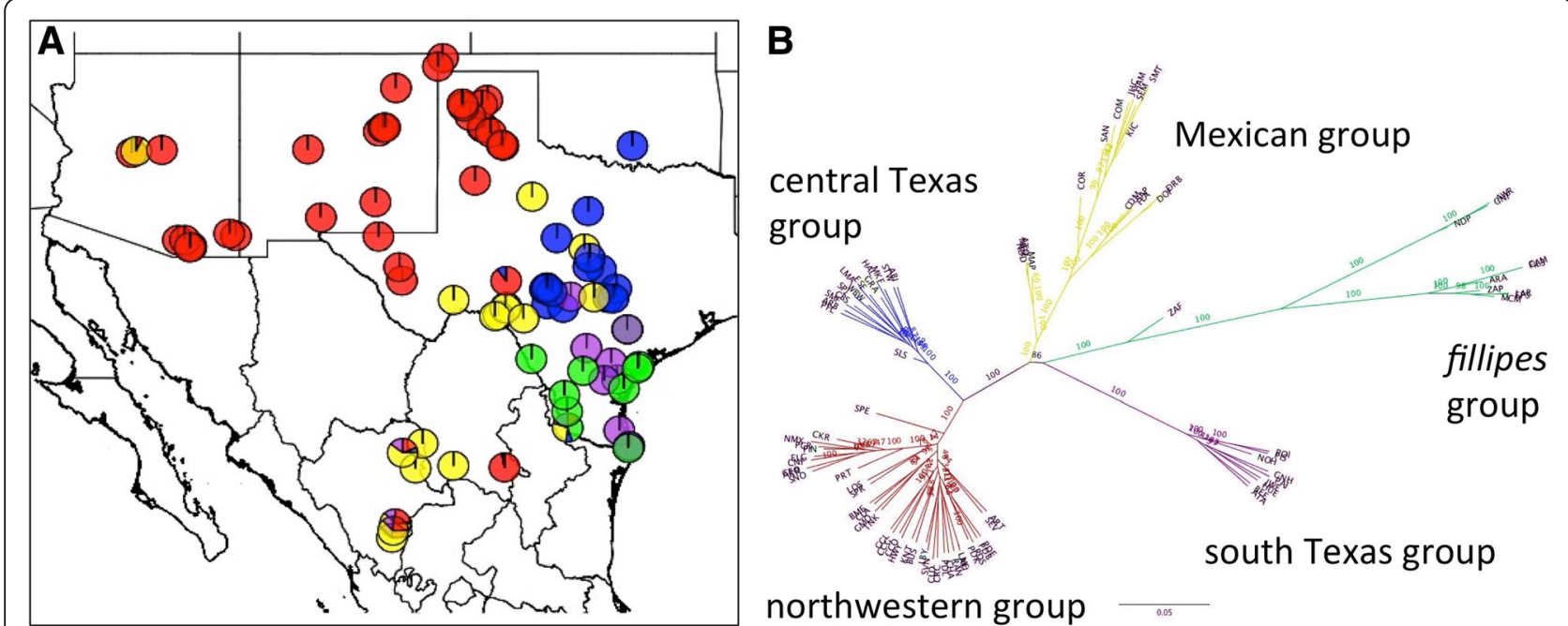

Fig. 1 Population structure of 89 P.hallii individuals. a Geographic sampling locations colored by proportion of STRUCTURE group membership. b RAxML phylogenetic tree. Major clades on the tree are colored to match the corresponding groups in (a)

been identified near Gonzalez, TX. The resequenced var. filipes and var. hallii lines from this population showed no evidence of mixed ancestry. In contrast, several individuals showed evidence of hybridization between groups within var. hallii. Seven of 78 var. hallii individuals showed significant evidence of mixed ancestry between one or more groups. All of these mixed individuals were collected from the southern part of the range (Fig. 1).

Geography is correlated with genetic distance across the range of P.hallii (Fig. 3). Correlation of genetic distance with geographic distance can arise due to limited interbreeding between geographically separated groups

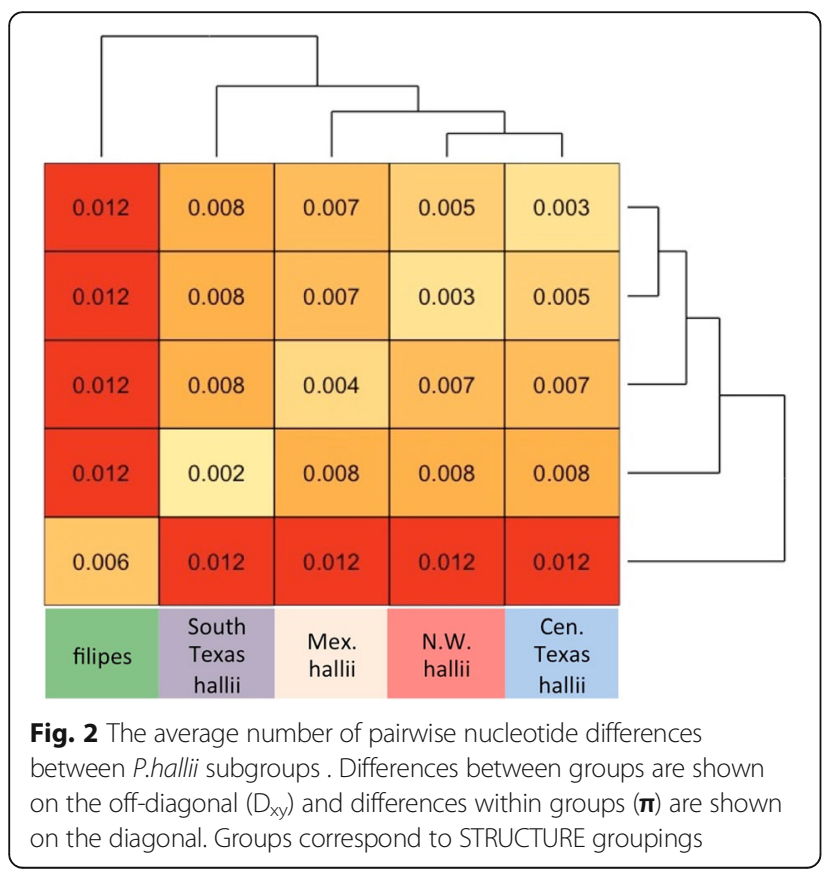

as well as due to hierarchical population structure [19]. In P.halii we found that overall isolation by geographic distance (IBD) was not significant across the entire $P$. hallii collection after controlling for the effects of population substructure (see Methods). However, IBD was strong and statistically significant within three of the

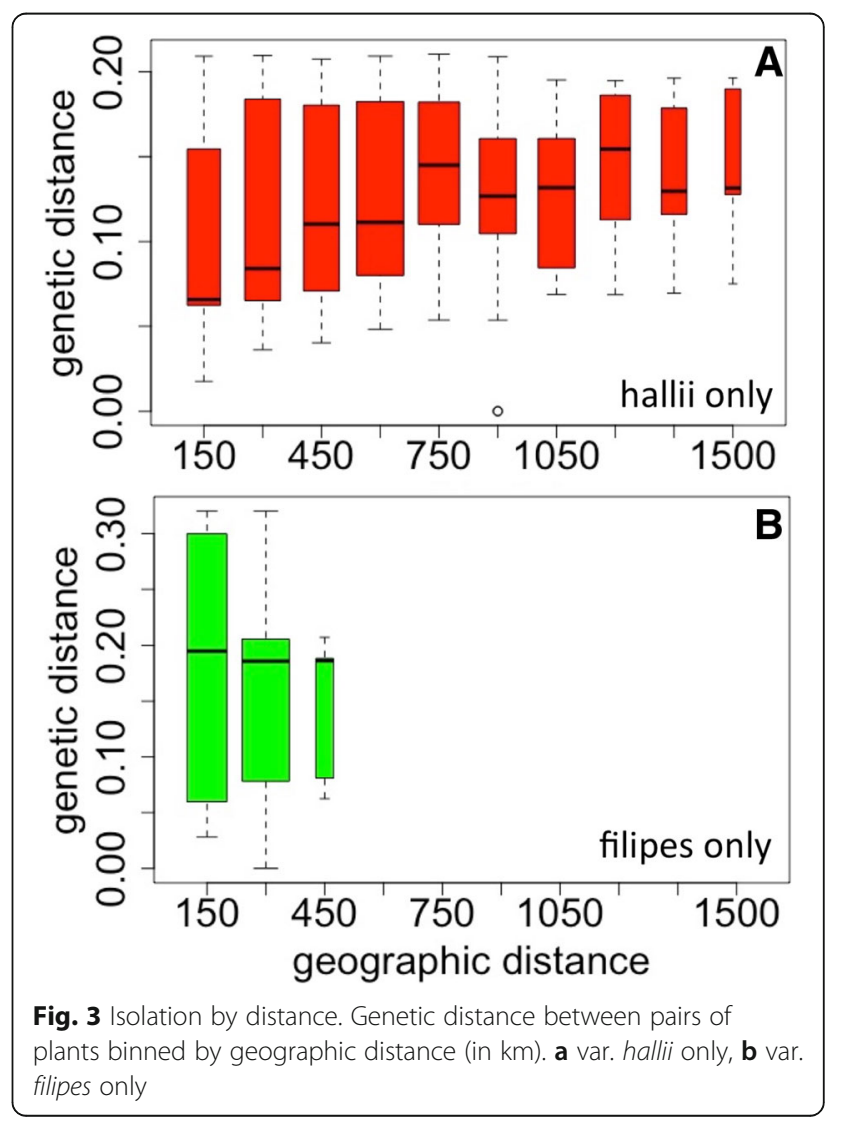


individual subgroups: Northwestern hallii (Fig. 1, red), Mexican hallii (yellow), and South Texas hallii (purple) (Additional file 2: Table S5). Although the NW hallii group spans the greatest geographic distance, IBD was less strong in this group (Mantel's $r=0.580$ ) than within the smaller South Texas group (Mantel's $r=0.728$ ). We hypothesize that genetic diversity across P.hallii is the result of some isolation by distance combined with hierarchical structure that developed as a result of expansion from southern glacial refugia following the last glacial maximum.

\section{Climate}

We examined variation in precipitation and temperature across the species range using data from 19 Bioclimatic variables from the WorldClim database (Additional file 1: Figures S3 and S4). The major contrasting axes of variation in precipitation across the range were most strongly correlated with annual mean precipitation, seasonality of precipitation, and mean precipitation in the warmest quarter (Additional file 1: Figure S3). Major variation in temperature was characterized most strongly by variation in mean daily temperature range, isothermality, and minimum temperature of the coldest month. Aridity, a measure of water stress imposed on plants due to the combined influence of solar radiation, precipitation and temperature, also varied significantly across the range. In general, climate in the study area varies across a northwest to southeast axis, from the hot dry deserts of Arizona, New Mexico, western Texas, and northern Mexico to the more mesic regions along the Gulf Coast (Additional file 1: Figure S5). Var. filipes is found only in the coastal part of the range in South Texas, whereas var. hallii is found throughout the study area with the exception of some parts of the Gulf Coast region (Fig. 1).

Population structure within $P$. hallii as a whole was correlated with many of the major climate variables we examined. Genetic distance between individuals, regardless of subspecies identity, was significantly correlated with differences in collection site climate including mean annual precipitation, precipitation seasonality, average precipitation in the warmest quarter, mean daily temperature range, minimum temperature of the coldest month, and aridity (all $P<0.01$, Table 1 ). Correlation of climate with genetic distance between individuals was strongest for minimum temperature and temperature daily range (both Mantel's $r=0.42$ ).

\section{Climate GWAS}

We conducted a genome-wide association study (GWAS) of high frequency (MAF > 10\%) SNPs against each major climate variable (Additional file 1: Figure S5). We repeated the analysis for all $P$. hallii individuals and for the set of only var. hallii plants (Additional file 1: Figure S6). We used the STRUCTURE population membership coefficients as a control for the correlation between climate and relatedness (see Methods). This method provided the strongest control for correlations between population structure and temperature variables with lesser control of correlations with precipitation (Additional file 1: Figure S5). Using Bonferroni corrected significance cut-offs we identified 139 unique SNPs significantly associated with climate across all plants and 170 SNPs associated with climate within var. hallii alone (Table 1). In both analyses the largest number of SNPs were associated with isothermality ( $N=51$ and 137 , respectively). While 41 SNPs were associated with aridity across the combined group, there were no SNPs associated with aridity in var. hallii alone, indicating this association may be primarily driven by differences between subspecies. Many climate associated SNPs in both analyses fell within genic or $1 \mathrm{~kb}$ promoter regions of the genome. In the analysis of all plants, for at least three climate variables - isothermality, minimum temperature of the coldest month, and aridity - associated SNPs were more often found in genes than expected by chance $(p<0.05$, Table 1$)$. In addition to falling within genic regions, the climate-associated SNPs were also weakly enriched for non-synonymous substitutions $(p=0.04)$ suggesting many may be functional in nature. To test for the effect of recent strong selection on genes containing climate-associated variants we calculated

Table 1 Associations between climate variables and genetic variation

\begin{tabular}{|c|c|c|c|c|c|c|}
\hline $\begin{array}{l}\text { Climate } \\
\text { Variable }\end{array}$ & Description & range & $\begin{array}{l}\text { Correlation with genetic } \\
\text { distance (Mantel's r) }\end{array}$ & $\begin{array}{l}\text { No. Associated } \\
\text { SNPS }\end{array}$ & $\begin{array}{l}\text { No. SNPS } \\
\text { in genes }\end{array}$ & $\begin{array}{l}\text { No. Genes } \\
\text { Affected }^{a}\end{array}$ \\
\hline BioClim3 & isothermality (daily/annual temperature range) & $\begin{array}{l}0.017- \\
0.029\end{array}$ & -0.04 & 51 & $32^{*}$ & 26 \\
\hline BioClim2 & mean daily temperature range (degrees $\times 10$ ) & 84-195 & $0.41^{* *}$ & 32 & 10 & 7 \\
\hline BioClim6 & minimum temperature of the coldest month (degrees C) & $2.20-5.32$ & $0.42^{*}$ & 12 & $12^{*}$ & 5 \\
\hline BioClim12 & annual mean precipitation (mm) & $220-953$ & $0.22^{* *}$ & 13 & 6 & 4 \\
\hline BioClim18 & precipitation in the warmest quarter (mm) & $99-271$ & $0.20^{*}$ & 1 & 1 & 1 \\
\hline Aridity & $\begin{array}{l}\text { mean annual precipitation/mean annual potential evapo- } \\
\text { transpiration }\end{array}$ & $\begin{array}{l}0.128- \\
0.706\end{array}$ & $0.24^{*}$ & 42 & $6^{*}$ & 4 \\
\hline
\end{tabular}

${ }^{\text {a }}$ The number of unique genes containing the snps associated with a climate variable

${ }^{*} p<0.05 ;{ }^{* *} p<0.01$ 
both variable-site nucleotide diversity $\left(\pi_{\mathrm{var}}\right)$ and Tajima's D. We found no evidence of reduced diversity or an excess of rare variants in climate-associated genes. Variable site $\pi$ was similar in climate and non-climate associated genes $\left(\pi_{\mathrm{var}}=0.163\right.$ and 0.161 , respectively). Tajima's $\mathrm{D}$ was close to zero and similar between the gene groups $(\mathrm{D}=-0.17$ and -0.21 , respectively).

Because there are strong correlations between climate and geography across the study area, we examined more closely how both factors influence variation in the candidate climate SNPs identified by GWAS. We used redundancy analysis (RDA) to calculate the proportion of variation in each set of candidate SNPs (for each climate variable) that can be explained separately by climate and by geography (Additional file 2: Table S4). For all sets of candidate SNPs the variation explained by the combination of climate and geography combined was greater than expected by chance (permutation tests $p<0.001$, Additional file 2: Table S4). For all sets of candidate SNPs, a greater percentage of variation could be explained by climate than by geography. Variation in minimum temperature of the coldest month (BioClim variable 6) explained the greatest percentage of variation in candidate SNPs $(41.9 \%, n=12$ SNPs). Geography explained only $5.1 \%$ of variation in the same SNP set. Most other climate variables including mean daily temperature, isothermality, annual precipitation and aridity explained between 12 and $15 \%$ of candidate SNP variation, geography explaining between 5 and $11 \%$ of variation (Additional file 2: Table S4).

Following genome-wide analyses, we examined the putative function of genes containing climate associated SNPs (Additional file 2: Tables S1 and S2). SNPs associated with aridity were in genes enriched for functions related to dormancy processes, seed maturation, response to hyperosmosis and salinity, and abscisic acid metabolism and biosynthesis. One SNP in Pahal.C02265, a gene associated with regulation of stomatal closure and transpiration in Arabidopsis [20], was correlated with both aridity and annual precipitation but fell just above the $p$-value cutoff used in this study $\left(p=2.0 \times 10^{-8}\right.$ for aridity and $p=$ $2.3 \times 10^{-8}$ for annual precipitation). SNPs in genes associated with daily temperature range were enriched for functions related to hormone metabolism and biosynthesis. Lastly, SNPs associated with isothermality were enriched for involvement in drought recovery. An isothermality associated SNP fell within Pahal.D02753. The Arabidopsis ortholog of this gene (AT1G70670) is a negative regulator in abscisic acid (ABA) signaling (Fig. 4; [21]).

\section{Discussion}

In this study, we were able to characterize genomic variation across geographic space for 89 accessions of the $\mathrm{C} 4$ perennial grass species, $P$. hallii. Consistent with a previous smaller-scale study [11], we found strong patterns of population structure between the two previously identified subspecies (varieties) in this system, var. hallii and var. filipes. We also found evidence that var. hallii is further partitioned into at least four other groups, which all have partially overlapping geographic ranges. Each of those groups may constitute a different ecotype (as defined by [6]), which may have evolved allopatrically and only recently come into secondary contact. To explore the extent to which climate and geography have shaped genomic variation in this species we used whole genome sequencing to assay over 9 million SNPs and identified many which are strongly associated with climate variation. We discuss these findings in more detail below.

\section{The structure of species complexes}

Population structure analysis of $P$. hallii revealed that it is partitioned into at least five subgroups. This revelation of complexity of population structure in $P$. hallii parallels recent findings in its close relative switchgrass $(P$. virgatum). The literature on switchgrass typically partitioned that species into upland and lowland ecotypes $[22,23]$, which have similar morphological differences as between var. hallii and var. filipes. However, just as in this study recent population structure analyses and new collections in the southern US have revealed that other major groups also exist within $P$. virgatum $[12,24]$.

Overall, the divided population structure of $P$. hallii and other plant species suggests that many widespread species exist as subspecies complexes, with groups at different stages of the speciation continuum occurring across their range [25]. The most divergent subgroups, var. hallii and var. filipes, are at least partially reproductively isolated. This idea is supported by Lowry et al. [15] who identified a two-locus Dobzhansky-Muller incompatibility in a cross between var. hallii and var. filipes. There may also be some reproductive isolation between the four subgroups identified within P. hallii in this study. Admixture between the varieties appears to occur at a relatively low rate, despite having overlapping geographic ranges. Future studies should quantify reproductive isolating barriers between these groups and sample more individuals within populations, especially those in sympatric regions, to establish the extent of gene flow between the various groups.

The patterns of population structure of $P$. hallii may also provide insights into general biogeographic divisions across its range. In particular, there is a strong break in population structure that runs diagonal through West Texas from northeast to southwest, (a break dividing the red and yellow population subgroups in Fig. 1a). The break is strongly associated with a transition from lower elevation in the east to higher elevation in the west. To the west of the break are the higher elevation populations of $P$. hallii, which primarily cluster as a single distinct 

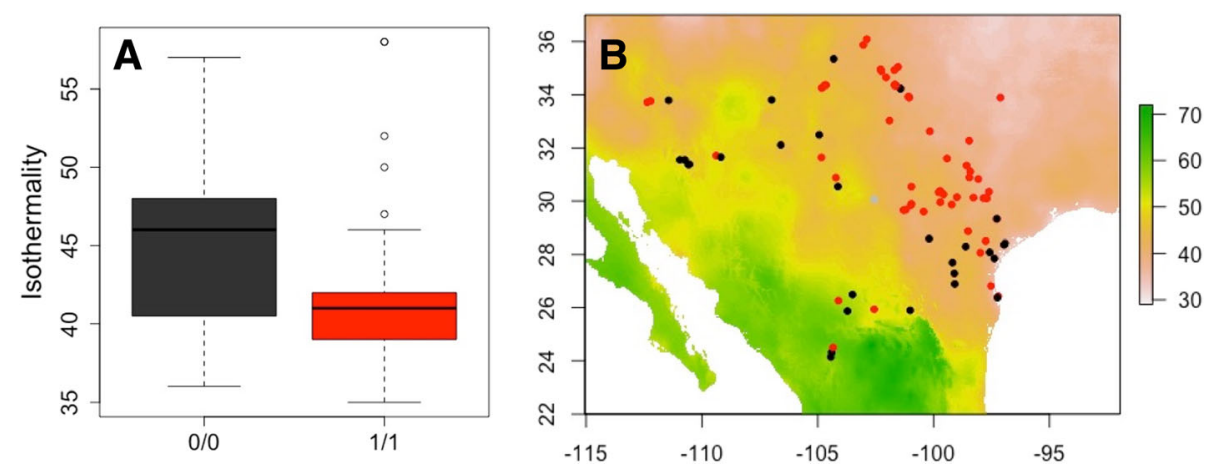

Fig. 4 Distribution of genotypes at a SNP in candidate gene Pahal.D02753 that is putatively involved in drought recovery and is associated with climate isothermality (daily/annual temperature range). a Home site isothermality values for homozygous genotypes at the SNP. b Geographic distribution of SNP genotypes. Map shading represents isothermality ratios. Homozygous reference allele in black, homozygous alternate allele in red, missing genotype in grey

group. Lowry et al. [11] found that the morphology of these high elevation populations is also distinct from the rest of $P$. hallii, as these plants have a larger overall size and have the largest seeds of any of the plants in the species complex. To the east of the break are all the other ecotypes of $P$. hallii, including var. filipes. This break in Texas corresponds to the transition from the gently rolling Edward/Stockton Plateaus to mountainous Trans-Pecos region in the south and the transition between the rolling lower plains to the high plains further north. These vegetation regions of Texas are well established [26, 27]. While few molecular population structure studies of plants have been conducted in this region, a recent study found a very similar ecogeographic split in Melampodium leucanthum in West Texas [28]. Multiple population genetic groups have also been identified in animals in this region, with the origins of these groups hypothesized to be the result of multiple Pleistocene refugia [29, 30].

The extent to which the geographic ranges of subgroups of $P$. hallii overlap may actually be underestimated by this study. In particular, the "Mexico" subgroup (Fig. 1, yellow) may be more widespread. One accession collected from a population in Arizona (COR) clustered with populations from central Texas and Mexico, just as it did in Lowry et al. [11]. The population exists at a relatively low elevation (1110 m.a.s.l.) compared to other collections in Arizona, New Mexico, and West Texas. This suggests the possibility that the Mexico subgroup is also distributed throughout the lower elevations of the desert Southwest of the United States.

\section{Climate associations}

Similar to studies of other plants, there is evidence that genetic distance is correlated with both geographic separation and major climate variables in this species. Despite confounding between climate and geography we were able to identify SNPs that were significantly associated with all major climate variables. These in turn are likely to be driving phenotypic variation that is correlated with environment. While variation in climate-associated SNPs was also associated with geography, more candidate SNP variation could be explained by climate than by geography in all cases.

It is unclear whether climate or isolation by distance has a stronger influence on genome-wide genetic similarity in natural plant populations, but at least one study in Arabidopsis [31] demonstrated an equal influence of both factors. We find climate associated SNPs are not often associated with more than one climate variable in $P$. hallii. This result parallels findings of Fournier-Level et al. [32], who found that while fitness associated SNPs in Arabidopsis had non-random distributions, they were not often associated with fitness in more than one environment.

In $P$. hallii climate associated SNPs were more often found in genes than expected by chance, which is similar to findings in [31, 33]. This suggests that the candidate genes we identified contain functional or regulatory changes that contribute to climate adaptive phenotypic differences between population groups. Genes containing climate-associated variants were only weakly enriched for non-synonymous changes, which contrasts somewhat with recent findings in natural populations of other species. We also found little evidence that genes containing climate associated SNPs have been under stronger directional or purifying selection than other genes in the genome. Climate SNPS also do not appear to have been subject to recent selective sweeps. In Arabidopsis and cultivated Sorghum, climate associated SNPs were often non-synonymous [31, 34] and in Brachypodium, more than half of climate-associated loci showed signs of recent selection [35].

The putative function of many loci associated with climate variation in $P$. hallii are linked to adaptive traits that are known or suspected to vary between locally adapted populations. Genes associated with both dormancy and 
response to hyperosmosis and salinity (both experienced during drought stress) contained climate associated SNPs. $P$. hallii is exposed to variable levels of drought stress throughout its range during the summer and becomes dormant in the winter months. Population subgroups, perhaps nascent ecotypes, would be predicted to have appreciable differences in both drought tolerance and avoidance response (dormancy). One candidate gene (Pahal.D02753) containing climate-associated variation is likely to be involved broadly with drought recovery through the regulation of the key stress hormone ABA.

\section{Conclusions}

Using population genomic sequencing and analyses we identified multiple population subgroups within the perennial C4 grass P.hallii. These population subgroups were partially overlapping across the species range and may represent early stages in the formation of subspecific ecotypes. Genomic variation across subgroups was significantly driven by adaptation to broad-scale climate variation in temperature, precipitation and aridity. Genes containing climate-associated SNPs most likely function in stress response regulation, tolerance, and recovery. These genes provide targets for further research into the molecular basis of adaptation along ecological gradients, particularly in perennial grass species.

\section{Methods}

\section{Plant collections and genome sequencing}

Many of the accessions used in this study were collected from field sites as seeds or live plants in the years 20102014. The authors thank the following for providing seed collections and permission for our collections: The Ladybird Johnson Wildflower Center, The Brackenridge Field Laboratory, The Nature Conservancy of Texas, The State Parks of Texas, The Kika de la Garza Plant Materials Center, members of Christine Hawkes laboratory, and The University of Arizona and Arizona State University Herbaria. Seeds were collected from private lands through permission granted by the Texas Ecolab project. Seeds were imported from Mexico by under a University of Texas herbarium permit, number SGPA/ DGVS/04170/14.

A single accession was sampled from each population to maximize sampling across the geographic range of $P$. hallii and to avoid biases introduced by strong pairwise among deme population structure [36]. Because only a single plant was sampled at each location, in this study we can draw inferences in relation only to range-wide patterns of genetic diversity and correlations with climate but not about within population variation in allele frequencies. Additional accessions were acquired from various existing seed collections (Additional file 2: Table S3). Collections were processed as described in Lowry et al. [11]. All accessions that were used in this study were grown from seed in the University of Texas at Austin greenhouses. High-quality DNA was extracted from leaf tissue using Qiagen DNeasy Plant Mini Kits (Qiagen, Hilden, Germany).

Whole genome sequencing was performed at two sequencing centers. Eighty lines were sequenced at the Joint Genome Institute (Walnut Creek, CA) on either the Illumina HiSeq2000 or HiSeq2500 platforms. The remaining libraries were sequenced at the University of Texas at Austin. Sequencing was paired-end at $150 \mathrm{bp}$ read length.

Short read data has been archived in the NCBI Short Read Archive (https://www.ncbi.nlm.nih.gov/sra). Accession numbers are listed in Additional file 2: Table S3.

\section{SNP calling and filtering}

Reads were aligned against the v2 draft P.hallii genome (filipes variety; FIL2 genotype, available at phytozome.org) using BWA MEM (v0.7.12) and the parameters (-M $-\mathrm{p}-$ t8). Before alignment, the genome was repeat masked for 24-mers occurring $>=650$ times. This masked approximately $5 \%$ of the genome. BAM files were screened for PCR duplicates and areas around indels were realigned using GATK IndelRealigner [37]. SNPs were called on the filtered alignments using the VarScan v2.4.1 mpileup2snp command [38] with a minimum coverage of eight reads and a minimum supporting read count of four to call SNPs. All SNPs within 25 bp of a repeat masked region or a gap were eliminated from the data set to eliminate low-quality SNP calls. Heterozygous calls were refined using a binomial exact test of read counts for each allele, with heterozygous calls excluded at a combined significance level of $p>0.05$. SNPs were only retained that had a greater than $90 \%$ call rate across all individuals and that had at least two individuals supporting each homozygous allele.

The reference genome line (FIL2) was included in the original sample set, and as a conservative control for sequencing or assembly errors calls at any site where an alternate allele was called in the re-sequenced FIL2 line were considered missing data $(N=15,714$ nucleotide positions). We also observed unusually high numbers of heterozygous calls in pericentromeric regions of the genome. This is likely due to inaccuracy of alignment in these areas, which are known to often be repeat-rich in plants $[17,18]$. The observed level of heterozygosity in P. hallii at microsatellite markers was about 5\% [11], so we eliminated all SNP calls with greater than 5\% heterozygous calls. We also eliminated SNPs with quality scores less than 40 , greater than $10 \%$ missing genotype calls, more than two alleles, and greater than +2 standard deviations read coverage (103 reads). The final data set contained 9,270,046 SNPs genotyped across 89 individuals. SNPs 
were annotated using SNPeff v4.1 k [39] and the v2.0 annotation of the P. hallii genome (www.phytozome.org).

\section{Analysis of population structure and linkage}

A set of synonymous SNPs were isolated for use in analyses of population structure. Using VCFtools v0.1.11 [40], we generated a set of synonymous variants that occurred only on major scaffolds and were no closer to one another than 3000 bp $(N=28,640)$ to control for possible linkage disequilibrium. We designate this set of markers as 'neutral SNPs'.

We analyzed the neutral SNPs with RAxML v8.0.6 [41] to generate an un-rooted phylogenetic tree reflecting the most likely relationships between individuals. Neutral SNPs were concatenated for each individual. We used a tree-building model with no rate heterogeneity and a correction for ascertainment bias (ASC_GTRCAT), with a maximum of 1000 bootstrap replicates. The resulting tree was visualized using FigTree (http:// tree.bio.ed.ac.uk/software/).

The neutral SNPs were also used to analyze population clustering with STRUCTURE [42]. We converted the SNP data to STRUCTURE format using the program PGDSpider [43] and then completed five runs each for values of $K=2-8$. Each run had a default 10,000 burn-in MCMC iterations and an additional 10,000 iterations following. Results for all runs were summarized using Structure Harvester [44] and plotted with Distruct [45]. The number of structured groups with the highest deltaK value [46] was 3. However, because the RAxML analyses identified at least five major clusters, we used five population clusters for downstream analyses.

Isolation by distance (IBD) was tested using a Mantel test, in the $\mathrm{R}$ package vegan, of the matrix of geographic distances between sampling locations vs. the matrix of genetic distances between individuals. Genetic distance between individuals was calculated as 1-(probability of identity by state) for neutral SNPs in TASSEL [47]. Geographic distances between sampling coordinates were calculated using the R package Imap v1.32 [48] . Significance of IBD was tested using 999 permutations stratified by subpopulation group membership. We also used Mantel tests to evaluate correlations between pairwise differences in collection site climate variables and pairwise genetic relatedness.

\section{Climate association analysis}

Full BioClim environmental data (19 variables) was downloaded for the study area from http://www.worldclim.org/ bioclim. Climate variable values were extracted for a set of 127 P.hallii known sampling locations using the data portal at http://dataportal-senckenberg.de/dataExtractTool/. To choose climate variables for association analysis, we used principal components analysis to determine the axes of greatest climate variation across the study area and to identify variables with the least amount of correlation. First, we determined the principal components of the set of all precipitation variables (bio12-bio19) and identified the individual variables that loaded most heavily on the first three principal components (Additional file 1: Figure S3). The variables of interest were bio12 (annual mean precipitation), bio15 (precipitation seasonality), and bio18 (amount of precipitation in the warmest quarter). Due to missing data values for a subset of the collection sites, bio15 was later dropped from the analysis. Second, we determined the principal components of the set of all temperature variables for the study area (bio1-bio11). The variables that loaded most heavily on the first three components were bio2 (temperature mean daily range), bio3 (isothermality = daily/annual range), and bio6 (minimum temperature of the coldest month). We also downloaded data on aridity for the region from the CGIAR-CSI global database (http://www.cgiar-csi.org/ data/global-aridity-and-pet-database). Aridity is calculated as mean annual precipitation divided by mean annual potential evapo-transpiration. Data for climate variables isothermality, minimum temperature of the coldest month, and precipitation in the warmest quarter were transformed to improve normality before analysis.

We used TASSEL to test the association between SNPs and each selected climate variable. To avoid bias we only analyzed SNPs with a minor allele frequency (MAF) of $10 \%$ or greater $(N=3,059,501)$. To control for population structure, we tested three linear models: one that incorporated a random effect kinship matrix, one that used the STRUCTURE population membership covariates ( $Q$ values), and one that used both [49]. Use of both $\mathrm{Q}$ coefficients and a kinship matrix together produced conservative results that over-corrected for population structure, evident as skewed $p$-value distributions. Because use of a model with $\mathrm{Q}$ coefficients alone provided stronger control for structure than using a K-matrix alone, we chose the former for all subsequent analyses. We used a Bonferroni corrected significance threshold of $p<1.63 \times 10^{-8}$ for associations. Enrichments for SNP position (genic/non-genic) and type (synonymous/non) were conducted using Fisher exact tests or the chisquared approximation in $\mathrm{R}$. We repeated the association tests within var. hallii alone, excluding all var. filipes individuals. In that analysis, there were 2,241,150 SNPs with MAF $>10 \%$. We used a Bonferroni corrected significance threshold of $p<2.23 \times 10^{\wedge^{-8}}$.

We tested whether regions of potentially low-quality alignment and SNP calling in peri-centromeric regions of the genome were likely to generate false positives. We used TASSEL to reanalyze only SNPs that fell outside of peri-centromeric regions. In general, climate associated 
SNPs in that analysis were not more or less often located in genic regions than for the full analysis. Because this is an indicator that SNP calling errors do not strongly contribute to error in the identification of climate associated SNPs, we present here results for SNPs in all regions of the genome.

\section{Redundancy analysis (RDA)}

RDA was performed on the set of significant candidate SNPs associated with each climate variable. To test the influence of geography on each candidate SNP set we used the first principal component (PC) of latitude and longitude of all plant collection sites as a predictor variable. Missing genotype values were imputed as heterozygous. Significance of each constrained axis of variation (climate variable and geographic PC) was measured using 999 permutation tests. RDA was executed using the vegan package in R [50]. For the single SNP associated with BioClim variable 18, we instead used linear regression of SNP variation on climate and geographic PC separately to measure the amount of variation explained as $R^{2}$ values.

\section{GO term enrichment analysis}

We tested for enrichment of gene-ontology (GO) terms among associated SNPs for each climate variable. To annotate GO terms, all P.hallii reference proteins were paired with their best Arabidopsis protein matches using BLAST (blastp) at an $e$-value cutoff of $10^{-3}$. This set of proteins (including duplicates) was used as the background gene set against which to test hypotheses of significant enrichment of GO terms for each gene set of interest. Subsequent GO enrichment tests for genes near climate associated SNPs were done using the R BIOCONDUCTOR package topGO [51]. We used the Bioconductor database 'org.At.tair.db' for annotation and the algorithm 'classic' (statistic = 'fisher') for statistical tests. We report GO terms with uncorrected enrichment $P$-values $<=0.05$ (Additional file 2: Table S1).

\section{Additional files}

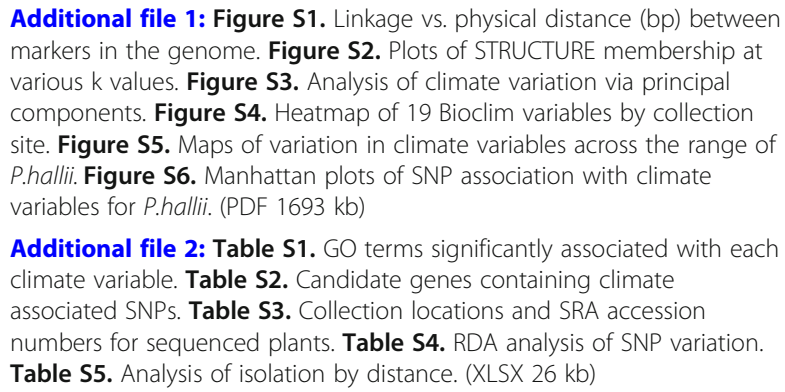
markers in the genome. Figure S2. Plots of STRUCTURE membership at various $k$ values. Figure S3. Analysis of climate variation via principal components. Figure S4. Heatmap of 19 Bioclim variables by collection site. Figure S5. Maps of variation in climate variables across the range of P.hallii. Figure S6. Manhattan plots of SNP association with climate variables for P.hallii. (PDF $1693 \mathrm{~kb}$ )

Additional file 2: Table S1. GO terms significantly associated with each climate variable. Table S2. Candidate genes containing climate associated SNPS. Table S3. Collection locations and SRA accession numbers for sequenced plants. Table S4. RDA analysis of SNP variation. Table S5. Analysis of isolation by distance. (XLSX 26 kb)

\section{Abbreviations}

ABA: Abscisic acid; BAM: Binary alignment; BioClim: Bioclimatic variables derived from monthly temperature and rainfall values in the WorldClim database (http://www.worldclim.org/bioclim); FIL2: Filipes line 2, the reference genome accession; GATK: Genome analysis toolkit (software, [37]); GWAS: Genome wide association study; IBD: Isolation by distance; MAF: Minor allele frequency; MCMC: Markov Chain Monte Carlo;

QTL: Quantitative trait locus; RAxML: Randomized Axcelerated Maximum Likelihood (software, [41]); RDA: Redundancy analysis; SNP: Single nucleotide polymorphism; TASSEL: Trait Analysis by aSSociation, Evolution and Linkage (software, [47]); TX: Texas

\section{Acknowledgements}

The authors thank A. Asmus, C. Purmal, T. Quedensley, J. Reilley, and S. Taylor for help with collections and plant propagation. A. Wrobleski assisted in preparation of the manuscript. We thank J. Lovell and $Y$. Brandvain for computational advice. We also thank the following for providing seed collections and/or permission for our collections: The Ladybird Johnson Wildflower Center, The Brackenridge Field Laboratory, The Nature Conservancy of Texas, The State Parks of Texas, The Kika de la Garza Plant Materials Center, members of Christine Hawkes lab, as well as The University of Arizona and Arizona State University Herbaria, Socorro González Director of the Herbarium at the Instituto Politécnico Nacional, Durango, México.

\section{Funding}

This research was supported by the U.S. Department of Energy, Office of Science, Office of Biological and Environmental Research award number DESC0014156 to TJ and through award number DE-FC02-07ER64494 (Great Lakes Bioenergy Research Center) to BG and DL. Funding was also provided by a National Science Foundation Plant Genome Research Program Award (IOS-0922457) to TJ, a United States Department of Agriculture NIFA-AFRI postdoctoral fellowship (2011-67012-30696) to DL, and a Linda Escobar Award from the Plant Biology Program at University of Texas at Austin to JP. Funding agencies played no role in the design of the study or collection, analysis, and interpretation of data or writing the manuscript. The work conducted by the U.S. Department of Energy Joint Genome Institute is supported by the Office of Science of the U.S. Department of Energy under Contract No. DE-AC02-05CH11231.

\section{Availability of data and materials}

Short read sequence data for all plant accessions from this study are available from the NCBI Short-Read Archive (https://www.ncbi.nlm.nih.gov/sra, see Additional file 2: Table S3 for accession numbers). Genotype calling results are available in VCF format and STRUCTURE group membership coefficients for all individuals from the authors upon request. Climate data used in this study were from the publicly available WorldClim database located at http://www.worldclim.org/bioclim.

\section{Authors' contributions}

$\mathrm{DL}, \mathrm{TJ}$, and JS devised the study. DL and JP collected seeds, grew plants, and extracted DNA. KB managed the JGI portion of the data collection, interfaced with the collaborators, and provided overall project management for sequencing of the $P$. hallii accessions. JJ and SM aligned read data and called genotypes. BG analyzed SNP data and conducted GWAS analyses. BG and DL wrote the manuscript. All authors read and approved the final manuscript.

Ethics approval and consent to participate

Not applicable.

\section{Consent for publication}

Not applicable.

\section{Competing interests}

The authors declare that they have no competing interests.

\section{Publisher's Note}

Springer Nature remains neutral with regard to jurisdictional claims in published maps and institutional affiliations.

\section{Author details}

'Myriad Women's Health, South San Francisco, CA 94080, USA. ${ }^{1}$ Department of Plant Biology, Michigan State University, East Lansing, MI 48824, USA. ${ }^{3}$ Great Lakes Bioenergy Research Center, Michigan State University, East Lansing, MI 48824, USA. ${ }^{4}$ Department of Integrative Biology, University of 
Texas at Austin, Austin, TX 78712, USA. ${ }^{5}$ Genome Sequencing Center, HudsonAlpha Institute for Biotechnology, Huntsville, AL 35806, USA. ${ }^{6}$ Department of Energy, Joint Genome Institute, Walnut Creek, CA 94598, USA. ${ }^{7}$ Plant Resilience Institute, Michigan State University, East Lansing, MI 48824, USA

\section{Received: 2 June 2018 Accepted: 17 October 2018}

Published online: 01 November 2018

\section{References}

1. Turesson $\mathrm{G}$. The genotypic response of the plant species to habitat. Hereditas. 1922;3:211-350.

2. Clausen J. Stages in the evolution of plant species. Ithaca: Cornell University Press; 1951.

3. Wadgymar S, Lowry DB, Gould BA, Byron CN, Mactavish R, Anderson JT. Identifying targets and agents of selection: innovative methods to evaluate the environmental and genetic factors that contribute to local adaptation. Methods Ecol Evol. 2017;8:738-49.

4. Linhart YB, Grant MC. Evolutionary significance of local genetic differentiation in plants. Annu Rev Ecol Syst. 1996;27:237-77.

5. Sobel JM. Ecogeographic isolation and speciation in the genus Mimulus. Am Nat. 2014;184:565-79.

6. Lowry DB. Ecotypes and the controversy over stages in the formation of new species. Biol J Linn Soc. 2012;106:241-57.

7. Gordon SP, Contreras-Moreira B, Woods DP, Des Marais DL, Burgess D, Shu S, Stritt C, Roulin AC, Schackwitz W, Tyler L, Martin J. Extensive gene content variation in the Brachypodium distachyon pan-genome correlates with population structure. Nat Commun. 2017;8:2184.

8. Roda F, Ambrose L, Walter GM, Liu HL, Schaul A, Lowe A, Pelser PB, Prentis P, Rieseberg LH, Ortiz-Barrientos D. Genomic evidence for the parallel evolution of coastal forms in the Senecio lautus complex. Mol Ecol. 2013;22(11):2941-52.

9. Burke SV, Wysocki WP, Zuloaga FO, Craine JM, Pires JC, Edger PP, MayfieldJones D, Clark LG, Kelchner SA, Duvall MR. Evolutionary relationships in Panicoid grasses based on plastome phylogenomics (Panicoideae; Poaceae). BMC Plant Biol. 2016;16(1):140.

10. Lowry DB, Purmal CT, Meyer E. Microsatellite markers for the native Texas perennial grass, Panicum hallii (Poaceae). Am J Bot. 2012:99:114-6.

11. Lowry DB, Purmal CT, Juenger TE. A population genetic transect of Panicum hallii (Poaceae). Am J Bot. 2013;100:592-601.

12. Lowry DB, Behrman KD, Grabowski P, Morris GP, Kiniry JR, Juenger TE. Adaptations between ecotypes and along environmental gradients in Panicum virgatum. Am Nat. 2014;183(5):682-92.

13. Gould FW. The grasses of Texas. College Station: Texas A\&M University Press; 1975

14. Waller FR. A biosystematic study of Panicum section Diffusa (Poaceae) in North America. Diss Abstr Int B. 1976:37(3):1094-5.

15. Lowry DB, Hernandez K, Taylor SH, et al. The genetics of divergence and reproductive isolation between ecotypes of Panicum hallii. New Phytol. 2015;205:402-14.

16. Lovell JT, Schwartz S, Lowry DB, Shakirov EV, Bonnette JE, Weng X, Wang M, Johnson J, Sreedasyam A, Plott C, Jenkins J, Schmutz J, Juenger TE. Drought responsive gene expression regulatory divergence between upland and lowland ecotypes of a perennial C4 grass. Genome Res. 2016;26(4):510-8.

17. Schnable PS, Ware D, Fulton RS. The B73 maize genome: complexity, diversity, and dynamics. Science. 2009;326:1112-5.

18. Schmutz J, McClean PE, Mamidi S, et al. A reference genome for common bean and genome-wide analysis of dual domestications. Nat Genet. 2014; 46(7):707.

19. Miermans PG. The trouble with isolation by distance. Mol Ecol. 2012;21:2839-46.

20. Kelly G, Sade N, Doron-Faigenboim A, Lerner S, Shatil-Cohen A, Yeselson Y, Egbaria A, Kottapalli J, Schaffer AA, Moshelion M, Granot D. Sugar and hexokinase suppress expression of PIP aquaporins and reduce leaf hydraulics that preserves leaf water potential. Plant J. 2017:91(2):325-39.

21. Kim YY, Jung KW, Yoo KS, Jeung JU, Shin JS. A stress-responsive caleosin-like protein, AtCLO4, acts as a negative regulator of ABA responses in Arabidopsis. Plant Cell Physiol. 2011:5:874-84

22. Porter CL. An analysis of variation between upland and lowland switchgrass, Panicum virgatum L., in central Oklahoma. Ecology. 1966:47:980-92.

23. Stroup JA, Sanderson MA, Muir JP, McFarland MJ, Reed R. Comparison of growth and performance in upland and lowland switchgrass types to water and nitrogen stress. Bioresour Technol. 2003;86:65-72.
24. Grabowski PP, Morris GP, Casler MD, Borevitz JO. Population genomic variation reveals roles of history, adaptation and ploidy in switchgrass. Mol Ecol. 2014;23(16):4059-73.

25. Lowry DL, Gould B. The speciation continuum. In: 'Encyclopedia of Evolutionary Biology'. R. Kliman, head editor, D. Ortiz-Barrientos, section editor. Elsevier, online publication; 2016. p. 159-65.

26. Bray WL. The ecological relations of the vegetation of western Texas. Bot Gaz. 1901;32(2):99-123.

27. Gould FW, Hoffman GO, Rechenthin CA. Vegetational areas of Texas. Leaflet/Texas Agricultural Extension Service; no. 492. 1960.

28. Rebernig CA, Schneeweiss GM, Bardy KE, Schoenswetter P, Villasenor JL, Obermayer R, Stuessy TF, Weiss-Schneeweiss HA. Multiple Pleistocene refugia and Holocene range expansion of an abundant southwestern American desert plant species (Melampodium leucanthum, Asteraceae). Mol Ecol. 2010;19(16):3421-43.

29. Riddle BR, Hafner DJ. A step-wise approach to integrating phylogeographic and phylogenetic biogeographic perspectives on the history of a core North American warm desert biota. J Arid Environ. 2006;66:435-61.

30. Castoe TA, Spencer CL, Parkinson CL. Phylogeographic structure and historical demography of the western diamondback rattlesnake (Crotalus atrox): a perspective on North American desert biogeography. Mol Phylogenet Evol. 2007;42:193-212.

31. Lasky JR, Des Marais DL, McKAY JO, Richards JH, Juenger TE, Keitt TH. Characterizing genomic variation of Arabidopsis thaliana: the roles of geography and climate. Mol Ecol. 2012;21(22):5512-29.

32. Fournier-Level A, Wilczek AM, Cooper MD, Roe JL, Anderson J, Eaton D, Moyers BT, Petipas RH, Schaeffer RN, Pieper B, Reymond M, Koornneef M, Welch SM, Remington DL, Schmitt J. Paths to selection on life history loci in different natural environments across the native range of Arabidopsis thaliana. Mol Ecol. 2013;22(13):3552-66.

33. Hancock AM, Brachi B, Faure N, Horton MW, Jarymowycz JB, Sperone FG, Toomajian C, Roux F, Bergelson J. Adaptation to climate across the Arabidopsis thaliana genome. Science. 2011;334(6052):83-6.

34. Lasky JR, Upadhyaya HD, Ramu P, Deshpande S, Hash CT, Bonnette J, Juenger TE, Hyma K, Acharya C, Mitchell S, Buckler ES, Brenton Z, Kresovich S, Morris GP. Genome-environment associations in sorghum landraces predict adaptive traits. Sci Adv. 2015;1(6):e1400218.

35. Dell'Acqua M, Zuccolo A, Tuna M, Gianfranceschi L, Pè M. Targeting environmental adaptation in the monocot model Brachypodium distachyon: a multi-faceted approach. BMC Genomics. 2014;15(1):801.

36. Stadler T, Haubold B, Merino C, Stephan W, Pfaffelhuber P. The impact of sampling schemes on the site frequency spectrum in nonequilibrium subdivided populations. Genetics. 2009;182:205-16.

37. McKenna A, Hanna M, Banks E, Sivachenko A, Cibulskis K, Kernytsky A, Garimella K, Altshuler D, Gabriel S, Daly M, DePristo MA. The Genome Analysis Toolkit: a MapReduce framework for analyzing next-generation DNA sequencing data. Genome Res. 2010:20:1297-303.

38. Koboldt DC, Chen K, Wylie T. VarScan: variant detection in massively parallel sequencing of individual and pooled samples. Bioinformatics. 2009;25:2283-5.

39. Cingolani P, Platts A, Wang LL, Coon M, Nguyen T, Wang L, Land SJ, Ruden DM, Lu X. A program for annotating and predicting the effects of single nucleotide polymorphisms, SnpEff: SNPs in the genome of Drosophila melanogaster strain w1118; iso-2; iso-3. Fly. 2012;6:1-13.

40. Danecek P, Auton A, Abecasis G, Albers CA, Banks E, DePristo MA, Handsaker RE, Lunter G, Marth GT, Sherry ST, McVean G, Durbin R. The variant call format and VCFtools. Bioinformatics. 2011;27:2156-8.

41. Stamatakis A. RAxML version 8: a tool for phylogenetic analysis and postanalysis of large phylogenies. Bioinformatics. 2014;30:1312-3.

42. Pritchard JK, Stephens M, Rosenberg NA. Association mapping in structured populations. Am J Hum Genet. 2000:67:170-81.

43. Lischer HEL, Excoffier L. PGDSpider: an automated data conversion tool for connecting population genetics and genomics programs. Bioinformatics. 2012;28:298-9.

44. Earl DA. STRUCTURE HARVESTER: a website and program for visualizing STRUCTURE output and implementing the Evanno method. Conserv Genet Resour. 2011:4:359-61.

45. Rosenberg NA. Distruct: a program for the graphical display of population structure. Mol Ecol Resour. 2003;4:137-8.

46. Evanno G, Regnaut S, Goudet J. Detecting the number of clusters of individuals using the software STRUCTURE: a simulation study. Mol Ecol. 2005; $14: 2611-20$. 
47. Bradbury PJ, Zhang Z, Kroon DE, Zhang Z, Kroon DE, Casstevens TM, Ramdoss Y, Buckler ES. TASSEL: software for association mapping of complex traits in diverse samples. Bioinformatics. 2007;23:2633-5.

48. Wallace JR. Interactive Mapping: Package 'IMAP'. version 1.32. 2015. https:// CRAN.R-project.org/package=Imap.

49. Zhang Z, Ersoz E, Lai CQ. Mixed linear model approach adapted for genome-wide association studies. Nat Genet. 2010:42:355.

50. Oksanen J, Blanchet FG, Friendly M, Kindt R, Legendre P, McGlinn D, Minchin PR, O'Hara RB, Simpson GL, Solymos P, Stevens MHH, Szoecs E, Wagner $\mathrm{H}$. vegan: Community Ecology Package. R package version 2.5-2. 2018. https://CRAN.R-project.org/package=vegan

51. Alexa A, Rahnenführer J. Gene set enrichment analysis with topGO. 2015 https://topgo.bioinf.mpi-inf.mpg.de/

Ready to submit your research? Choose BMC and benefit from:

- fast, convenient online submission

- thorough peer review by experienced researchers in your field

- rapid publication on acceptance

- support for research data, including large and complex data types

- gold Open Access which fosters wider collaboration and increased citations

- maximum visibility for your research: over $100 \mathrm{M}$ website views per year

At BMC, research is always in progress.

Learn more biomedcentral.com/submissions 